\title{
Methodological problems with the use of the retroactive interference design to infer what is stored
}

\author{
KEITH N. CLAYTON and MICHAEL W. WARREN \\ Vanderbilt University, Nashville, Tennessee 37240
}

\begin{abstract}
The retroactive interference paradigm has been used in a variety of settings to investigate the nature of the representation of memory. Much of the research using this paradigm is methodologically flawed because it involves treatment comparisons which are inappropriate. It is argued that comparisons must be made between presentation conditions having the same interpolated activity and that, in addition, evidence is required that the differences are not confounded by acquisition level differences if one is to infer mode-specific interference and representation. The methodological issues are discussed in detail and the utility of the retroactive interference design is questioned. Studies employing the design are reviewed, and several conclusions are drawn: (1) there has been no unambiguous demonstration of visual mode-specific interference, (2) there has been no clear demonstration that imagery instructions produce memories that are more susceptible to visual than to auditory interpolation, and (3) no clear demonstrations are yet available that memory for spatial location is more susceptible to visual interference than memory for letters.
\end{abstract}

The retroactive interference design has been employed in the study of memory in order to obtain evidence related to the representation issue, that is, the issue of what is "stored." The assumption of the paradigm is that intervening activities interfere with the memory when either (a) the representation of the activity resembles the representation of the memory, or (b) the activity and memory compete for the same limited capacity system. Hopkins, Edwards, and Cook (1973), for example, found that recall of auditorially presented items was impaired more by auditorially presented than by visually presented interpolated activity. The result was used to support a claim that auditorially and visually presented materials are represented in separate stores. The retroactive interference design has been used to infer differential coding or storage for a variety of experimental conditions: auditory vs. visual presentation (e.g. . Hopkins et al., 1973), concrete vs. abstract verbal stimuli (e.g., Sasson \& Fraisse, 1972), imaging vs. verbal repetition instructions (e.g., Elliot, 1973), pictures vs. words (e.g., Wells, 1972), and identity vs. spatial location in a visual display (e.g., Meudell, 1972), etc. We will claim that much of the research in these areas has involved inappropriate comparisons between the wrong experimental conditions, and, therefore, has prompted conclusions which could be incorrect. In short, this paper is addressed to some methodological difficulties that emerge when the activities intervening between presentation and recall are manipulated with the intent of inferring how to-be-remembered material is represented in memory.

In the following paragraphs, we intend: (a) to examine the methodological issues in detail, (b) to illustrate the errors that have been made, as well as validate our assertion that the errors are widespread, and (c) to make recommendations about appropriate comparisons, as well as specify the conditions which are essential for the appropriate use of the retroactive interference design.

\section{COMPONENTS ANALYSIS OF RETROACTIVE INTERFERENCE}

It is convenient to discuss the retroactive interference design within the framework of a hypothetical experiment. Suppose we wish to contrast the memories for materials presented in two acquisition modes. These modes may be sensory modalities, instruction conditions, or involve stimulus variables, etc. There are six possible treatment conditions produced by combining factorially two acquisition modes with three interpolation conditions (the two modes plus a control condition). The rows of Table 1 illustrate the six conditions, assuming the modes are the auditory and visual modalities. The six conditions are designated $\mathrm{AA}, \mathrm{AV}, \mathrm{VA}, \mathrm{VV}, \mathrm{AC}$, and $\mathrm{VC}$, where the first letter refers to presentation modality and the second refers to the intervening conditions. The purpose of the hypothetical study is to determine whether there is modality-specific interference, that is, whether the interpolated activity is interfering, in part at least, because it is in the same modality as the acquisition condition. Modalityspecific interference will be interpreted as evidence for modality-specific memorial representation, in this case, differential coding or storage of auditory and visual memory items. The columns of Table 1 are 
Table 1

Components Analysis of Retroactive Interference Present in Six Conditions of Experiment Involving Auditory (A) and Visual (V) Presentations

\begin{tabular}{|c|c|c|c|c|c|c|}
\hline \multicolumn{2}{|c|}{ Conditions } & \multicolumn{5}{|c|}{ Components } \\
\hline \multirow{2}{*}{$\begin{array}{l}\text { Acqui- } \\
\text { sition } \\
\text { Mode }\end{array}$} & \multirow{2}{*}{$\begin{array}{l}\text { Inter- } \\
\text { polated } \\
\text { Activity }\end{array}$} & \multicolumn{3}{|c|}{ Nonspecific } & \multicolumn{2}{|c|}{$\begin{array}{l}\text { Modality } \\
\text { Specific }\end{array}$} \\
\hline & & $\mathbf{A}$ & $\mathrm{V}$ & $\mathrm{C}$ & A & $\mathbf{V}$ \\
\hline A & $\mathbf{A}$ & Yes & No & No & Yes & No \\
\hline A & V & No & Yes & No & No & No \\
\hline V & $\mathbf{A}$ & Yes & No & No & No & No \\
\hline V & V & No & Yes & No & No & Yes \\
\hline $\mathbf{A}$ & $\mathrm{C}$ & No & No & Yes & No & No \\
\hline $\mathrm{V}$ & $\mathrm{C}$ & No & No & Yes & No & No \\
\hline
\end{tabular}

Note-A "Yes" in a cell indicates hypothetical presence of the retroactive interference component listed in the column title. " $C$ " is the control, or no interpolated activity, condition.

labeled with the components of retroactive interference (RI) that are assumed to be present in the treatment conditions: nonspecific RI and modalityspecific Rl. Nonspecific RI may be thought of as the loss in retention due to the information processing demands of the interpolated activity (cf. Posner, 1966). Modality-specific RI is the hypothetical additional interference incurred when an interpolated task is presented in the same mode as the to-be-remembered material. If auditory-specific RI is present. only one condition. AA, should be affected by it. Similarly, only VV should be affected by visual-specitic $\mathrm{RI}$.

The critical issue to which the present paper is addressed is the question of which treatment comparisons are appropriate in order to properly evaluate the presence of modality-specific interference. The problem is that in addition to this potential for generating modality-specific interference, interpolated tasks also differ in the degree to which they prevent rehearsal (Keppel, 1965; Peterson, 1969) and in their potential for producing nonspecific interference (Posner, 1966). It is assumed that a particular auditory (or verbal or abstract, etc.) interpolated task can produce a different amount of nonspecific RI than the identical visual (or imaginal or concrete, etc.) interpolated task. For example, if the interpolated task requires digit categorization, then the auditory categorization task may be more suitable for preventing rehearsal or may require a different amount of cognitive effort, etc., than the visual categorization task and control conditions. The analysis in Table 1 reflects these possibilities by indicating a difference between auditory, visual, and control nonspecific RI.

Now we are prepared to evaluate the possible treatment comparisons. Consider, for example, a typical experiment in which the question is asked whether cross-modal interference is greater than intramodal interference. This usually is assessed by comparing conditions with the same presentation mode but different interpolations. This amounts to comparing AA with AV or VV with VA. But if the components analysis presented here is correct. these comparisons are inappropriate. Condition AA differs from AV in two respects: (a) AA has modality-specific interference, $\mathrm{AV}$ does not; and (b) AA has auditory nonspecitic interference. AV has visual nonspecific interference. Similarly, the comparison between VV and VA is inappropriate because they also differ in two ways. Thus, if a difference is found with these comparisons, the experimenter cannot tell whether it is due to the presence of modality-specific interference or due to a difference in nonspecific RI for the interpolated activities; he cannot tell whether it is because the two modalities are represented differently or whether the auditory and visual tasks produce different interference for other reasons.

Inspection of Table 1 reveals that the appropriate comparisons to make in order to evaluate modality-specific interference are between conditions involving the same interpolated task. Thus a test for the presence of auditory-specific interference would involve a comparison between AA and VA. In terms of the components of retroactive interference, these two conditions differ only with regard to the presence, if any, of the auditory-specitic component. Thus, if a difference is found between AA and VA, it cannot be due to contamination produced by a difference in nonspecitic interference. For similar reasons, a comparison between $\mathrm{AV}$ and $\mathrm{VV}$ is the appropriate test for visual-specific interference. However, while these comparisons avoid one problem, they introduce another. Since performance on the retention test should be intluenced by the level of original training. it follows that memory tested after AA might prove inferior to that tested after VA, merely because the auditory task was not as well learned initially as the visual. Similarly, a comparison between $V V$ and $A V$ could prove contaminated by a difference in original acquisition level. These considerations prompt an additional requirement to unambiguously demonstrate mode-specific interference, namely, some demonstration that the obtained difference is not due to a difference existing at the beginning of the retention interval. Assuming this analysis is correct, we are now prepared to summarize the requirements needed in order to conclude that evidence has been obtained for mode-specific interference: the comparison must be made between conditions having the same interpolated activity, and there must be additional evidence that the differences are not confounded by acquisition level differences.

\section{Controlling Degree of Original Learning}

Before reviewing the studies that have used the retroactive interference design. some general consideration must be given to the problem of 
acquisition level differences. One way to obtain evidence on the possibility of differences in degree of original learning would be to augment the experimental design with immediate test conditions. The basic design for evaluating mode-specific interference in a particular mode would then be a 2 by 2 factorial, one factor being presentation mode and the other being retention interval (an immediate test vs. a delay filled with interpolation in the appropriate mode). There are two general problems with this strategy, however. First, if performance is very near perfect on the immediate test, a failure to find a difference between the two presentation modalities may be due to a ceiling effect (Underwood, 1964). Second, a difference may be apparent on the immediate test, in which case the results are confounded. If the retention functions from unequal initial levels converge or diverge, the results would be ambiguous. Diverging functions would be expected even if the interpolated activity were not differentially interfering, because the conditions starting at the higher level would be expected to be forgotten more slowly. Converging functions would also be ambiguous if the delayed condition produced no differences because of possible basement effects or becaluse a difference in initial acquisition level masked mode-specific interference. On the other hand, not all results are uninterpretable if differences exist at the end of acquisition. One interpretable outcome would be wo retention functions that cross over. That is. if the presentation mode producing the higher initial level is remembered more poorly after interpolation (provided the interpolation is in the same mode as the presentation mode producing the higher initial level), the results can be interpreted safely as due to mode-specific interference. Of course, in this case. the interference would have to have been extensive enough to overcome the original level difference.

A second technique for evaluating the degree of original learning would involve comparing control conditions having the same delay duration as the experimental conditions. This approach is subject to the same pitfalls as the design incorporating immediate tests. That is, ceiling effects in the control conditions could mask significant acquisition level differences, or differences in the control conditions could compromise the scope of the experiment, as mode-specific representation could only be inferred for the presentation condition with the higher acquisition level, and then only if the appropriate crossover were observed. Of course, the absence of ceiling effects and significant differences in the control conditions would yield an unconfounded and uncompromised experiment.

From the discussion so far, it should be obvious that the strategy of augmenting the retroactive interference design with immediate test or delayed control conditions protects the experimenter from ill-founded inferences. However, in adapting this approach, the experimenter gambles that control condition ceiling effects and differences will not comprou use his experiment. Rather than gamble that presentation conditions will be learned to the same degree, a better approach might be to attempt to control for degree of original learning (Underwood, 1954. 1964). For short-term memory experiments, Keppel (1965) has mentioned two techniques for achieving this outcome: (a) varying presentation rates to equate initial acquisition levels, and (b) varying the number of units to be remembered to equate initial acquisition levels. While these techniques may help equate foi originai degree of iearning in some paradigms, their utility for the retroactive interference design is somewhat dubious. Both techniques necessarily require altering the available time for coding items between presentation conditions. That is, one condition will have more coding time per item than the other condition. This additional time may enable subjects to recode items along other dimensions. Consider the hypothetical case in which we are attempting to equate auditory and visual presentation for initial degree of learning by slowing the rate of visual presentation. A decline in the visual presentation rate might enable subjects to recode visual stimuli along verbal dimensions. We might then fail to detect visual-specific interference because visual stimuli were no longer represented solely in a visual code. In short. techniques for equating for original degree of learning may alter the way in which to-be-remembered items are coded and remembered. In view of all these complexities, we believe that where pilot work indicates that acquisition levels differ, the use of the retroactive interference design is contraindicated.

\section{REVIEW OF STUDIES USING THE RETROACTIVE INTERFERENCE DESIGN}

The published studies fall into two groups: those that incorporated all the experimental conditions required to make the appropriate comparisons and those that did not. Those failing to incorporate the needed conditions are reviewed first. beginning with two studies on the representation of memory for phrases and sentences.

\section{Studies Lacking Appropriate Conditions}

Sasson (1971) presented subjects with concrete sentences. Prior to recall, one experimental group viewed a set of pictures unrelated to the original sentences and the control group spent the same amount of time on a dot-estimation task. The experimental group subsequently recalled fewer of the sentences than did the control group. This result was replicated by Sasson and Fraisse (1972), who concluded that "concrete sentences are stored 
primarily, although not entirely, in the form of images" (p. 419). If the analysis presented above is correct, this conclusion is unwarranted because viewing pictures and estimating dots may produce different interference for any task.

Crowder and Morton (1969) developed the stimulus suffix and response prefix paradigms in conjunction with their evaluation of a model of memory for acoustic information. The technique involves presentation of a redundant stimulus at the end of a stimulus sequence (stimulus suffix) or requiring output of a redundant response at the beginning of recall of the sequence (response prefix). Research reviewed by Crowder and Morton (1969) has shown that recall of an auditorially presented list is differentially affected by an auditory suffix and a vocal prefix. Relative to a control condition, the suffix and prefix conditions produce poorer recall, the suffix having its greatest effect on the last item, the prefix affecting recall throughout the list. But the comparisons of the prefix and suffix conditions with the control conditions are equivalent to the AA vs. AC comparisons described above and do not permit an unambiguous assessment of modality-specific interference. Crowder and Morton (1969) also report an experiment which shows that auditory prefixes and suffixes do not produce different recall from control conditions when the list is presented visually. From these two sets of results, they conclude that auditory lists have an auditory representation whereas visual lists do not. But the appropriate comparison involves auditory and visual lists learned to the same degree and followed by the same interpolated activity. An experiment by Morton and Holloway (1970) is similarly flawed.

G. Cohen (1972) used the suffix paradigm to investigate the representation of memory for pictures. Subjects were given sequences of pictures of familiar objects, and in some conditions the sequences were terminated with redundant items (stimulus suffixes). In one condition, the stimulus suffixes were pictures, and in another, they were words. In comparison with a control condition which had no specific interference, the suffix groups showed poorer performance in recall of the latter portions of the list. The picture suffix condition also yielded better performance than the other two conditions in recall of the early items of the list. Cohen concludes: "the interaction of serial position with type of interference forces the conclusion that the memory code cannot be uniform over the entire series" (1972, p. 45). But the conclusion is not forced at all. as the picture and word suffix and control conditions may produce differential interference for reasons other than their modal differences. In this case, for example, the difference between the no-interference and suffix conditions on recency probably stems from the fact that the suffixes prevented maintenance rehearsal of the last few items whereas the control condition did not (cf. Craik \& Lockhart, 1972, p. 680). The superior primacy exhibited by the picture suffix condition requires another explanation, but it does not force the conclusion Cohen reaches.

Scarborough (1972) presented lists of digits auditorially followed by lists of letters or digits presented visually. After the interpolated activity, the subjects were asked to recall either the first or second list. We are concerned here only with the retroactive interference part of the Scarborough experiment, hence in the recall of the auditory lists, but the experiment serves to remind us that the components analysis presented here may readily extend to proactive designs as well. In any case, Scarborough found that recall of the auditory lists after visual interpolated activity did not differ from recall of the auditory lists after no interpolated activity. This comparison is equivalent to a comparison of $\mathrm{AV}$ vs. $\mathrm{AC}$ in Table 1, in which we see the conditions differ in two ways. But Scarborough's conclusion is uniquely dubious. Since no significant differences were obtained between $A V$ and $A C$. he concludes the auditory and visual material are independently stored. In this case, not only is the experimental design inadequate to test the hypothesis, but the conclusion of independent visual and auditory stores depends on an inability to reject the null hypothesis.

A slightly different problem exists with the interpretation of results obtained by Deutsch (1970) while examining memory for the pitch of tones. In this experiment. subjects were presented with a short tone of one pitch followed later by a yes-no recognition test for pitch. There were two conditions of interpolated activity, the presentation of six tones or the presentation of six numbers of equal loudness. Recognition performance was substantially worse after tone interpolation than after number interpolation. Despite the patent reasonableness of a position that tones and numbers have different memory representations, this experiment cannot permit an unambiguous conclusion. The possibility exists that the greater interference produced by the interpolated tones had nothing to do with the fact that they were in the same mode as the acquisition task. Deutsch did have two other control conditions, but both involved number interpolation and have no bearing on the problem discussed here. What is required is a demonstration that tones interfere with memory for tones more than with memory for numbers when degree of original learning of tones and numbers is equated.

In several studies which incorporated inappropriate comparisons, experimenters have acknowledged some awareness that interpolated activities may differ in ways other than their potential for generating modality-specific interference. However, the various attempts to handle the problem are not all 
satisfactory. In some cases, performance on the interpolated tasks is assessed and the assumption is made that nonspecific interference is equated if performance on one interpolated task is indistinguishable from that on another. For example, in two studies on the memory for visual geometric figures, $\mathrm{R}$. L. Cohen and Granstrom (1968, 1970) manipulated the modality (auditory or visual) of interpolated learning tasks. Performance on the interpolated tasks was indistinguishable, but the visual task interfered more with some measures of retention of the geometric figures than did the interpolated auditory task. But does indistinguishable performance on the interpolated tasks ensure equal nonmodal interference? Sullivan and Turvey (1974) found that an interpolated auditory arithmetic (AAT) task interfered less than an interpolated visual arithmetic task (VAT) with the memory for the order of tactile stimulation. They also found no difference in performance on the interpolated tasks. Does this permit a conclusion that tactile memory shares processes more common to vision than to audition? Sullivan and Turvey thought so, but they were careful to admit the possibility that "while performance was equivalent in the two conditions, the effort needed to achieve that level of performance in the VAT condition [could have been] greater than that needed in the AAT condition" (p. 605).

\section{Studies Incorporating Appropriate Comparisons}

In the next set of studies, the appropriate comparisons were available. usually because acquisition and interpolated activity were simultaneously manipulated. Frequently, however, the authors focus on other comparisons or there are difticulties with degree of original learning. We will treat these studies in two groups: (a) those in which degree of original learning is potentially confounded. and (b) those in which degree of original learning is not confounded and unambiguous conclusions can be drawn. Confounded studies are considered first, and wherever possible studies examining similar problems are grouped. Considered first are studies concerned with imagery and imagery instructions. In a study of memory for phrases, Atwood (1971) presented three groups with phrases designating an imaginary scene which the subjects were instructed to visualize; another three groups were presented abstract phrases with instructions to contemplate their meaning. Each phrase required $1 \mathrm{sec}$ to present and was followed for separate groups by $2 \mathrm{sec}$ of a visual, auditory, or control task. Comparing the groups which received the imaginal phrases. Atwood found poorer recall when the interpolated task was visual than when auditory. Among the groups receiving the abstract phrases, the auditory interpolation produced poorest recall. Atwood argues that the visual interpolated activity interfered more with imaginal concrete sentences, because the imagery mnemonic involves the visual system. But the comparisons made are inappropriate; they are equivalent to comparing $A V$ with $\mathrm{AA}$ and VV with VA, as discussed above. Statistical comparisons of the control groups were not made by Arwood, but it appears that the imaginal phrases were learned to a higher level than were the abstract phrases. We might take this into account while making the appropriate comparisons (between tasks) for the Atwood data, but the groups which received imaginal phrases also received a different number of phrases than the groups which received the abstract phrases. The logical entanglements seem hopeless for this data. Interpretation of a similar study by Elliot (1973) is also difficult. In this study, subjects were given triads of high-or low-imageable nouns, with either imagery or rote repetition instructions followed either by auditory or visual or control distracting tasks. An immediate test was given in the experiment, but recall was nearly perfect, so the appropriate comparison. which Elliot did not make, would be ambiguous. In a simpler study, Wells (1972) showed recall of pictures to be superior to recall of words after $30 \mathrm{sec}$ of verbal interpolated activity. This is the appropriate comparison, but an immediate test showed nearly perfect recall of both pictures and words, so we cannot tell whether the picture superiority at $30 \mathrm{sec}$ is due to verbal interpolated activity interfering more with the words, or whether there was better initial learning of the pictures.

Salthouse (1974) had subjects recall either the identity or locations of letters in stimulus arrays after either a visual or control interpolated task. The appropriate comparison can be made here, and after visual interpolated activity recall of letter locations is poorer than recall of letter identity, but the results of the control groups suggest that letter identity acquired a higher degree of original learning.

Meudell (1972) also compared memory for identity of letters in a visual array vs. memory for locations in the array. A critical difference, however, is that whereas Salthouse (1974) used a single retention interval. Meudell tested after 3, 9, or $30 \mathrm{sec}$ of an auditory task (counting backwards by threes) or a visual (vigilance) task. In this section, we consider the effect of the visual interpolated activity in the Meudell experiment. In this case, recall of letters was superior to recall of locations after $3 \mathrm{sec}$ as well as after $30 \mathrm{sec}$. Since these results can be accounted for by differences in degree of original learning, it cannot be concluded that letters are less susceptible than locations to visual interpolated activity. Murray and Newman (1973) also used several retention intervals in their comparison of the memory for identity vs. location of simple geometric shapes in a visual array when interfered with by counting aloud, drawing lines, copying arrows, or some combinations of those activities. Murray and Newman wish to conclude that location is 
coded visually and shape is coded verbally, but the appropriate comparisons are contaminated by possible original learning differences. A nointerference control group was also used but was not helpful, since performance was near perfect throughout all retention intervals.

den Heyer and Barrett (1971) offer a unique attempt to solve for possible differences in degree of original learning. They compare memory for location vs. identity of letters in matrices after visual and verbal interpolation. A no-interpolation condition was included, and it allowed the inference that identity information was recalled better than location information when there was no interpolation. To correct for this difference, den Heyer and Barrett express the results of the experimental conditions in terms of a percentage loss relative to the appropriate control. That is, the control group identifies the $100 \%$ level and the experimental results are expressed as a percent of that. This may be a fruitful area to explore, but the particular solution offered seems unsatisfactory, as it makes the dubious assumption that $x \%$ loss of a strong memory is equivalent to $x \%$ loss of a weak one, for all values of $x$.

Hopkins, Edwards, and Cook (1973) had subjects recall animal-name triads presented auditorially and visually in a Peterson-Brown task. The interpolated activity was presented auditorially or visually, and it was found that cross-modal interference was lower than intramodal interference. The authors concluded that auditory and visual information are "encoded differently" (p. 336), but this conclusion is based on inappropriate comparisons and the appropriate comparisons are made ambiguous by possible differences in original learning.

The shadow paradigm provides a comparison of retention for auditorially vs. visually presented verbal items following an auditory interpolated activity, specifically, voicing items presented over headphones. The reliable finding with this paradigm is greater delayed recall of visual input than of auditory input. However, several studies utilizing this paradigm are flawed by immediate-recall ceiling effects (Kroll, Parks, Parkinson, Bieber, \& Johnson, 1970; Parkinson, Parks, \& Kroll, 1971; Salzberg, Parks, Kroll, \& Parkinson, 1971). Results of a similar study by Margrain (1967) are unclear as only a single retention interval was employed.

So far we have considered only those experimental results which prompted conclusions that we argue are suspicious because of a possible confound with degree of original learning. We now turn to those experiments in which appropriate comparisons can be made and no confound is evident. Two such studies involve the shadow paradigm. Specifically, Parkinson (1972) and Kroll, Parkinson, and Parks (1972) showed significantly superior recall of the auditory lists after 0 or $1 \mathrm{sec}$ of shadowing, and significantly superior recall of visual lists after $20 \mathrm{sec}$ of shadowing. This is the crossover result described above; thus, these two studies seem to provide a clear demonstration of auditory mode-specific interference.

A similar crossover result was reported in the experiment on letter identity vs. letter location in the experiment by Meudell (1972) discussed above. Specifically, Meudell found that, after $3 \mathrm{sec}$ of counting backwards (the auditory task), recall of the letters was superior to recall of the locations. But at $30 \mathrm{sec}$, the reverse was true; locations were recalled better than letters. Although these particular comparisons are not evaluated statistically by Meudell, it seems saife to conclude that the faster forgetting of the letters than of the locations when the interpolated activity was counting backwards could not be due to an initial superiority of location memory since, if anything, the reverse was true. It therefore seems safe to conclude that the auditory interpolated activity provided more retroactive interference for the letter than for the location recall.

Wicker and Holley (1971) contrasted memory for pictures and words under conditions similar to the Atwood (1971) study discussed above, namely, each stimulus was followed by $4 \mathrm{sec}$ of a visual, auditory, or no interpolated activity. Wicker and Holley report that "visual distraction has a greater effect with pictorial than with verbal stimuli." Since the picture stimuli were better recalled than the word stimuli after no interpolated activity. it seems unreasonable to argue that the poorer recall after visual interpolation is due to superior initial learning of the word stimuli. However, the finding that recall of words was poorer than recall of pictures after auditory interpolation could be due to initial strength differences and may not be due to mode-specific interference.

\section{CONCLUSIONS}

Although the major aim of the present paper has been to discuss methodological issues stemming from the use of the retroactive interference design, we can conclude with a summary of the findings which, by the present account, are not confounded. First, in comparing auditory and visual presentation modality, we have seen two studies (Kroll, Parkinson, \& Parks, 1972; Parkinson, 1972) that provide a demonstration of auditory mode-specific interference, but no studies have yet unambiguously demonstrated visual mode-specific interference. Comparing picture vs. word recall, one study (Wicker \& Holley, 1971) has shown pictures to be differentially affected by visual interpolated activity relative to words. But we have located no clear demonstration that imagery instructions produce memories that are more susceptible to visual than to auditory interpolation. One study (Meudell, 1972) shows that memory for letters is more susceptible to verbal interference than 
memory for spatial location of letters, but no clear demonstrations are yet available that memory for location is more susceptible to visual interference than memory for letters.

\section{REFERENCES}

Atwood, G. An experimental study of visual imagination and memory. Cognitive Psychology, 1971, 2, 290-299.

COHEN, G. Serial position effects in the recall of picture sequences. The Quarterly Journal of Experimental Psychology, 1972. 24. $41-47$.

Cohen, R. L., \& Granstrom, K. Interpolated task and mode of recall as variables in STM for visual figures. Journal of Verbal Learning and Verbal Behavior, 1968, 7, 653-648.

Cohen, R. L., \& Granstrom, K. Reproduction and recognition in short-term visual memory. The Quarterly Journal of Experimental Psychology, 1970, 22, 450-457.

Craik, F. I. M., \& LockharT, R. S. Levels of processing: A framework for memory research. Journal of Verbal Leaming and Verbal Behavior, 1972, 11. 671-684.

Crowder, R. G.. \& Morton, J. Precategorical acoustic storage (PAS). Perception and Psychophysics, 1969, 5, 365-373.

DEN HEYER, K.. \& BarRetT, B. Selective loss of visual and verbal information in STM by means of visual and verbal interpolated tasks. Psychonomic Science, 1971, 25, 100-102.

Deursch, D. Tones and numbers: Specificity of interference in immediate memory. Science, 1970, 168, 1604-1605.

Ellior, L. Imagery versus repetition encoding in short and long-term memory. Journal of Experimental Psychology, $1973,100.270-276$.

ENGLE, R. W. The modality effect: Is PAS responsible? Journal of Experimental Psychology. 1974, 102, 824-830.

Hopkins, R. H., Edwards, R. E., \& Cook, C. L. Presentation modality. distractor modality, and proactive interference in short-term memory. Journal of Experimental Psychology, 1973, 98. 362-367.

Keppel. G. Problem of method in the study of short-term memory. Psychological Bulletin, 1965, 63, 1-13.

Kroll, N. E. A. Short-term memory and the nature of interference from concurrent shadowing. The Quarterly Journal of Experimental Psychology, 1972. 24, 414-417.

Kroll. N. E. A., \& Kellicurt, M. H. Short-term recall as a function of covert rehearsal and of intervening task. Journal of Verbal Learning and Verbal Behavior, 1972, 11, 196-204.

Kroll. N. E. A., Parkinson, W. R., \& Parks. T. E. Sensory and active storage of compound visual and auditory stimuli. Journal of Experimental Psychology, 1972, 95, 32.38.

Kroll. N. E. A., Parks, T., Parkinson. S. R., Bieber, S. L., \& Johnson, A. L. Short-term memory while shadowing: Recall of visually and of aurally presented letters. Journal of Experimental Psychology, 1970, 85, 220-224.

Margrain, S. A. Short-term visual memory as a function of input modality. The Quarterly Journal of Experimental Psychology. 1967. 19. 109.114.
Mevdell, P. R. Short-term visual memory: Comparative effects of two types of distraction on the recall of visually presented verbal and nonverbal material. Joumal of Experimental Psychology, 1972, 94, 244-247.

Morton, J., \& Holloway, C. M. Absence of a cross-modal "suffix effect" in short-term memory. The Quarterly Journal of Experimental Psychology, 1970, 22, 167-176.

MurRay, D. J., \& Newman, F. M. Visual and verbal coding in short-term memory. Journal of Experimental Psychology, $1973,100,58-62$.

Parkinson, S. R. Short-term memory while shadowing: Multipleitem recall of visually and of aurally presented letters. Journal of Experimental Psychology. 1972, 92, 256-265.

Parkinson. S. R., Parks. T. E., \& Kroll, N. E. A. Visual and auditory short-term memory: Effects of phonemically similar auditory shadow material during the retention interval. Journal of Experimental Psychology, 1971, 87, 274-280.

Penny, C. G. Order of report and interference effects in fourchannel bisensory memory. Canadian Journal of Psychology, 1974, 28, 371-382.

Peterson. L. R. Concurrent verbal activity. Psychological Review, 1969, 76, 376-386.

Posner. M. I. Components of skilled performance. Science, 1966. 152, 1712-1718.

Salthouse. T. A. Using selective interference to investigate spatial memory representations. Memory and Cognition, 1974. 2. 744.757 .

Salzberg. P. M., Parks, T. E., Kroll, N. E. A., \& Parkinson, S. R. Retroactive effects of phonemic similarity on short-term recall of visual and auditory stimuli. Joumal of Experimental Psychology, 1971, 91, 43-46.

SASSON, R. Interfering images at sentence retrieval. Joumal of Experimental Psychology, 1971, 89, 56-62.

SASSON, R. Y.. \& Fraisse, P. Images in memory for concrete and abstract sentences. Journal of Experimental Psychology. 1972, 94, 149-155.

SCarborough, D. L. Memory for brief visual displays of symbols. Cognitive Psychology, 1972, 3, 408-429.

Sullivan, E. V.. \& Turvey, M. T. On the short-term retention of serial. tactile stimuli. Memory and Cognition. 1974, 2.601-606.

UNDERWOOD. B. J. Speed of learning and amount retained: A consideration of methodology. Psychological Bulletin, 1954, 51. 276-282.

UNDERWOOD. B. J. Degree of learning and the measurement of forgetting. Journal of Verbal Learning and Verbal Behavior. 1964, 3. 112.129.

WELLS, J. E. Encoding and memory for verbal and pictorial stimuli. The Quarterly Journal of Experimental Psychology, 1972, 24, 242.252.

WICKER, F. W.. \& Holley, F. M. Distraction modality and stimulus modality in paired-associate learning. Psychonomic Science, 1971, 25, 218-220.

(Received for publication April 24, 1975; revision received July 14,1975 .) 